\title{
Continued Intercostal Branches Block in MidAxillary Line (BRILMA) as pain control technique for unilateral and severe thoracic Trauma patients with Non-Invasive Mechanical Ventilation
}

\author{
Gomez Martin A. ${ }^{1}$, García González N. ${ }^{2}$, Gutiérrez González C. ${ }^{2}$, Álvarez Arias C. ${ }^{2}$, González \\ Humbreiro J.A. ${ }^{3}$, Posada Mesa L.F. ${ }^{1}$ \\ ${ }^{1}$ Hospital San Agustín, Aviles, Spain, ${ }^{2}$ Hospital Universitario Central de Asturias, Oviedo, Spain
}

\section{BACKGROUND:}

Multiple trauma is the leading cause of mortality in people under 40 years in our region. Thoracic trauma (pulmonary contusion, multiple rib fractures, flail chest, Mechanical Ventilation (MV) related Pneumonia...) represent between 35-45\% of the morbidity and mortality. The introduction of Non-invasive Mechanical Ventilation (NIMV) improves mortality rates associated with chest trauma, but severe pain makes the establishment of these techniques difficult.

\section{GOAL OF STUDY:}

Carry out a project in order to observe and evaluate the continuous pain control by Intercostal Branch Block in MidAxillary Line (BRILMA) in patients with severe chest trauma (contusion / multiple rib fractures, flail chest) in need of restoration NIMV therapy.

\section{MATERIALS AND METHODS:}

Observational study from May-September 2016, 53 patients with serious multiple trauma. We collected age, sex, ISS, SAPS3, associated injuries with hemodynamic/neurological compromise, VAS at rest, VAS with inspiration after NIMV application and after BRILMA catheter placement with Ropivacaine $0.2 \%$ infusion up to $10 \mathrm{ml} / \mathrm{h}$.

\section{RESULTS:}

$\checkmark 39$ of 53 patients admitted in ICU with multiple injuries. Polytrauma patients with severe head injury, chest trauma and/or hemodynamic instability requiring Invasive MV (IMV), were discarded. Only 6 of the 14 patients remaining, required NIMV (42\%).

$\checkmark$ Patients are mostly men with average age of 32 years. Patients requiring NIMV have lower index of gravity (ISS and SAPS3) bearing no associated lesions (severe craneal injury, multiple fractures with hemodynamic compromise,...).
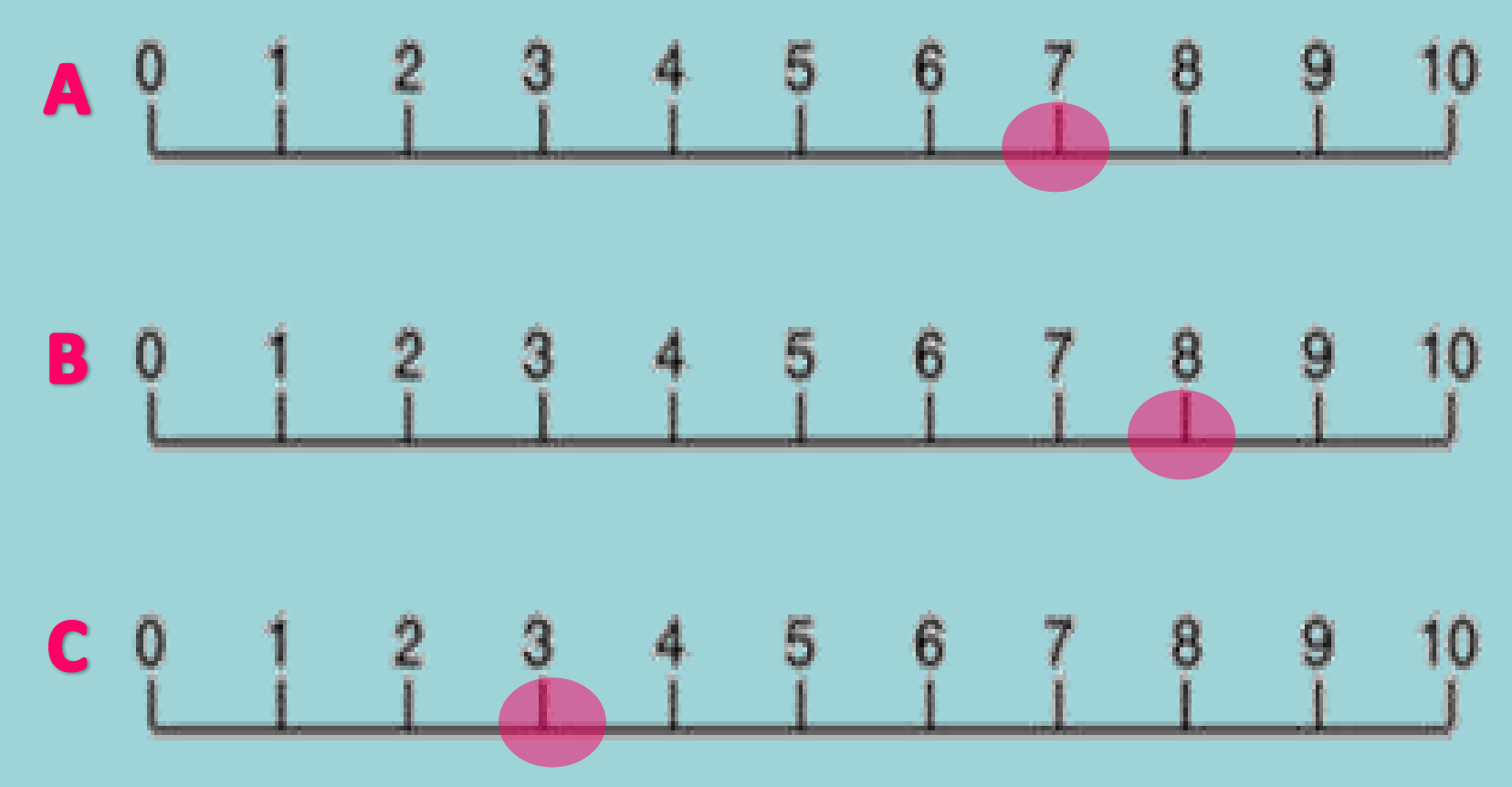

$\checkmark$ At rest, average values of VAS were close to $7+/-2(A)$. After introduction of NIMV, increased values of VAS $8+/-1.4$ without statistical significance $(B)$. We observed the decreasing in values of VAS after BRILMA block, with average values of $3+/-1.7(C)$ and this remains stable within the next 24 hours, with EVA average values of $3^{\prime} 5+/-2(D)$.

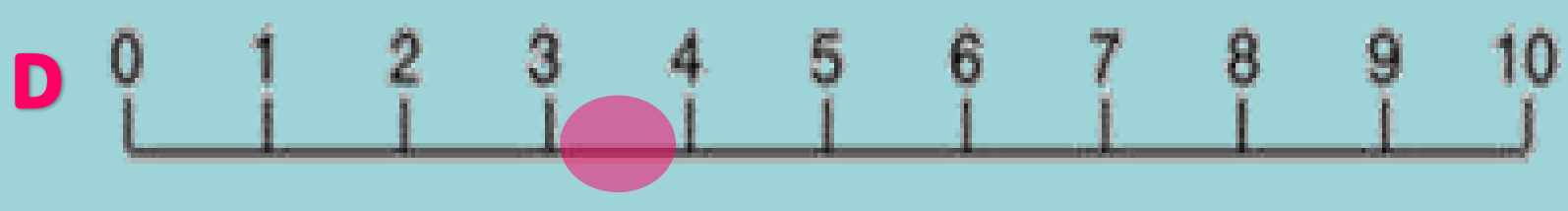

Visual Analog Scale (VAS)

\section{CONCLUSIONS:}

Continuous BRILMA block is an effective pain control technique in patients with unilateral thoracic trauma requiring NIMV therapy.

There are two key points: control of respiratory function and patient analgesia, so optimization and individualization based on injury and severity is compulsory.

The introduction of NIMV contributes to lower rates of morbidity and mortality so the proper pain control allows better tolerance of NIMV, avoiding IMV and its adverse consequences. 